American Journal of Infectious Diseases 8 (2): 73-78, 2012

ISSN 1553-6203

(C) 2012 Science Publications

\title{
Efficacy of an Oral Health-Promoting Program in Human Immunodeficiency Virus-Infected Children: A 7-year Longitudinal Study
}

\author{
${ }^{1}$ Nathalia Ferraz Oliscovicz, \\ ${ }^{1}$ Gloria Fernanda Barbosa de Araújo Castro, \\ ${ }^{2}$ Luciana Pomarico, ${ }^{1}$ Ivete Pomarico Ribeiro de Souza, \\ ${ }^{1}$ Department of Pediatric Dentistry and Orthodontics, \\ School of Dentistry, Federal University of Rio de Janeiro, Brazil \\ ${ }^{2}$ Department of Specific Formation, School of Dentistry, \\ Federal Fluminense University, Nova Friburgo, Brazil
}

\begin{abstract}
Problem statement: Children infected with HIV have a higher prevalence of caries in both dentitions. With that in mind, it is important the introduction of programmes for the control of caries disease in this population. Thus, this study was aimed to assess the efficacy of an oral healthpromoting program in controlling caries in HIV-infected children after 7 years of follow-up. Approach: Data on the oral health of 31 children were gathered and analyzed over a 7 -year period. Caries indexes were obtained by clinical examinations performed periodically by welltrained practitioners. An Epi Info software was used for analysis using the Mann-Whitney test. Results: DMFT index increased from 2.00-4.38, whereas deft index decreased from 5.19-4.31 $(p>0.05)$. By analyzing carious teeth, it was observed that the mean number of active carious teeth decreased $(P=0.001)$, whereas the mean number of inactive ones increased $(P=0.001)$. The mean extent of inactive caries was found to be greater in those cases in which the child attended more than 19 appointments $(\mathrm{P}=0.005)$. Conclusion: Based on such a decrease in active caries, one can conclude that the program was effective in improving the oral health of HIV-infected children.
\end{abstract}

Key words: Child, dental caries, HIV infections, child health services

\section{INTRODUCTION}

The first case of HIV infection in a child was reported in 1983, soon after the disease had been identified in adults. HIV infection has been spreading since, becoming a significant cause of mortality among children (Exposito-Delgado et al., 2004). In 2007, it was estimated that 2.5 million children worldwide under 15 were infected with HIV (WHO, 2006) and according to the most recent epidemiological bulletin, there are 15.775 children under 13 with AIDS in Brazil (Saúde, 2011).

Children infected with HIV have a higher prevalence of caries in both dentitions (Chen et al., 2003; Gelbier et al., 2000) compared to healthy counterparts (Madigan et al., 1996; Maguire et al., 1996; Ribeiro et al., 1998; Tofsky et al., 2000; Valdez et al., 1994). In addition, HIV-infected children show greater immunological impairment and thus are more likely to have higher caries activity (Castro et al., 2004). Several factors may be involved, such as carbohydrate-rich diet for calorie/protein reposition, high intake of sugar- containing compounds (Madigan et al., 1996; Maguire et al., 1996), decreased salivary flow (Ribeiro et al., 1998), low immune response to cariogenic bacteria (Madigan et al., 1996), inadequate oral hygiene and decreased salivary antibodies (Castro et al., 2004).

Because of their systemic condition, therefore, these children represent a special risk for early caries development as several factors affecting caries become part of their daily routine. Some of these factors can be controlled through oral health education. Of course, this does not apply to systemic conditions such as low immune response (Castro et al., 2004) or carbohydraterich diet. Considering all these factors, we believe that clinical exams and regular fluoride applications, as well as other interventions for promoting oral health, can effectively control the onset and progression of caries in HIV-infected children.

To test this hypothesis, the present study has assessed 7-year longitudinal data on caries indexes and caries activity in both deciduous and permanent dentitions of HIV-infected children taking part in an oral health program.

Correspondence Author: Luciana Pomarico, Department of Pediatric Dentistry and Orthodontics, School of Dentistry,

Federal Fluminense University, Nova Friburgo, Brazil 
Methods: The sample consisted of HIV-infected children (CDCP, 1994) between 2 and 14 years nonrandomly selected from a patient population attending the pediatric AIDS outpatient clinic of a public university hospital in Rio de Janeiro, Brazil. After their caregivers had given informed consent, the children participated in an oral health-promoting program by attending at least 9 appointments over a period of 7 years. The study was approved by the local ethics research committee.

Data on personal information, hygiene habits, presence of oral manifestations and dental caries were obtained from the patients' dental records. Medical history, lab results from the last 3 months (CD4, CD8) and use of medication were gathered from their medical records.

The program involved dental care routinely performed in the outpatient medical clinic while the patient awaited consultation with the pediatrician. The dentist brushed each patient's teeth with fluoride dentifrice, then applied topical $1.23 \%$ acidulated phosphate fluoride. In addition, the caregivers were instructed about oral hygiene. It should be emphasized that whenever the patient attended a medical consultation, he or she was also seen by a dentist in the program.

Oral examination was carried out by a well-trained practitioner using artificial light with the child lying on a stretcher. The program had 3 examiners over the 7year period of study; the kappa coefficients for intrarater reliability were, respectively, 0.92, 0.94 and 0.92 for examiners 1,2 and 3 . The examiner was positioned directly behind the child's head and carried out the examination using an intraoral mirror, a \#5 blunt-tipped probe and gauze to dry dental surfaces. After examining the soft tissues, each dental surface was investigated and classified according to a 0-9 score based on caries diagnostic criteria established by Carvalho et al. (1992) and Bjorndal et al. (1997) for, respectively, enamel and dentin (Fig. 1). After the exam, any cavitated lesion detected was then treated by using an atraumatic restorative technique
(Phantumvanit and Pilot, 1994) or extractions were performed when indicated. Patients were submitted to clinical reexamination every 3 months at least for following up caries incidence. Based on these dental records, it was possible to obtain the total number of active and inactive lesions and the caries indexes deft/defs and DMFT/DMFS (WHO, 1997), both modified to include the early enamel caries.

Data were entered into Epi Info software (CDC, version 3.2.2), then statistically analyzed using the Mann-Whitney test.

\section{MATERIALS AND METHODS}

The sample consisted of HIV-infected children (Centers for Disease Control and Prevention, 1996) between 2 and 14 years nonrandomly selected from a patient population attending the pediatric AIDS outpatient clinic of a public university hospital in Rio de Janeiro, Brazil. After their caregivers had given informed consent, the children participated in an oral health-promoting program by attending at least 9 appointments over a period of 7 years. The study was approved by the local ethics research committee.

Data on personal information, hygiene habits, presence of oral manifestations and dental caries were obtained from the patients' dental records. Medical history, lab results from the last 3 months (CD4, CD8), and use of medication were gathered from their medical records.

The program involved dental care routinely performed in the outpatient medical clinic while the patient awaited consultation with the pediatrician. The dentist brushed each patient's teeth with fluoride dentifrice, then applied topical $1.23 \%$ acidulated phosphate fluoride. In addition, the caregivers were instructed about oral hygiene. It should be emphasized that whenever the patient attended a medical consultation, he or she was also seen by a dentist in the program.

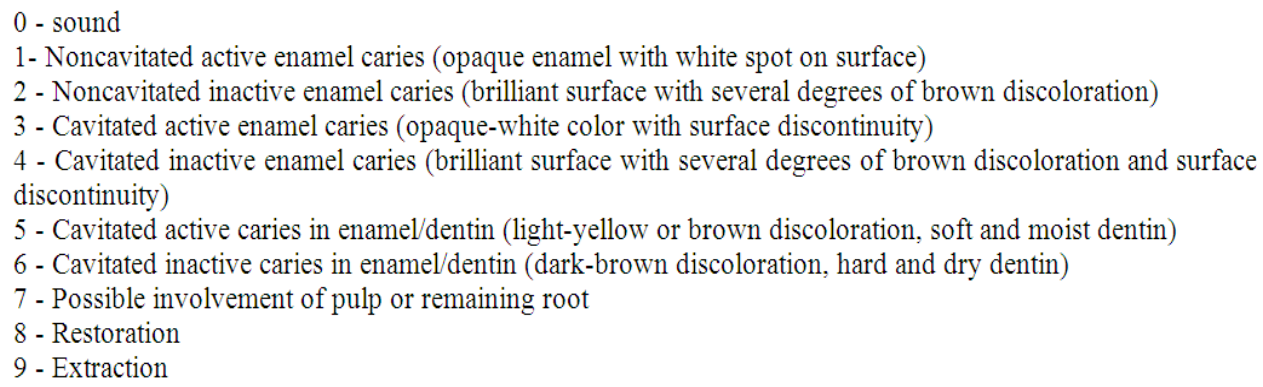

Fig. 1: Scores of dental examination in HIV-infected children Sources: Carvalho et al. (1992) and Bjorndal et al. (1997) 
Oral examination was carried out by a well-trained practitioner using artificial light with the child lying on a stretcher. The program had 3 examiners over the 7year period of study; the kappa coefficients for intrarater reliability were, respectively, 0.92, 0.94, and 0.92 for examiners 1,2 , and 3 . The examiner was positioned directly behind the child's head and carried out the examination using an intraoral mirror, a \#5 blunt-tipped probe, and gauze to dry dental surfaces. After examining the soft tissues, each dental surface was investigated and classified according to a 0-9 score based on caries diagnostic criteria established by Carvalho et al. (1992) and Bjorndal et al. (1997) for, respectively, enamel and dentin (Fig. 1). After the exam, any cavitated lesion detected was then treated by using an atraumatic restorative technique (Phantumvanit and Pilot, 1994) or extractions were performed when indicated. Patients were submitted to clinical reexamination every 3 months at least for following up caries incidence. Based on these dental records, it was possible to obtain the total number of active and inactive lesions and the caries indexes deft/defs and DMFT/DMFS (WHO, 1997), both modified to include the early enamel caries.

Data were entered into Epi Info software (CDC, version 3.2.2), then statistically analyzed using the Mann-Whitney test.

\section{RESULTS}

Thirty-one children had fulfilled the inclusion criteria, with a mean age of 5.29 years $(\mathrm{SD}=2.03)$ at baseline and 11.12 years $(\mathrm{SD}=2.13)$ at the end of the study. Of these patients, 19 were girls (61.3\%).

The analysis involved those values of dental caries found during the program every 2 years, namely: at baseline, after 2 years, after 4 years and at the end of the study. One can observe a variation in the caries indexes after 7 years, with decreases in deft and defs indexes and increases in DMFT and DMFS, although the differences were not statistically significant. As to the presence of carious lesions only, one can note a small, nonsignificant end-of-study (Table 1). Figure 2 shows the mean values for caries indexes deft and DMFT according to mean age at baseline, year 2, year 4 and final evaluation.

From the data, one can observe that the mean number of teeth presenting active caries (dentin and/or enamel) had decreased significantly, whereas there was a statistically significant increase in mean inactive caries (Fig. 3).

No statistically significant differences were found between caries indexes, number of carious lesions and presence of active and inactive caries in relation to sex $(\mathrm{P}>0.05)$.
Attendance in the program was based on the total number of appointments during the 7 years. The mean number of appointments was 19.32 (range 9-32). There was no relationship between sex and number of appointments as girls and boys had, on average, 19.15 and 19.58 appointments, respectively $(\mathrm{P}>0.05)$.

Attendance influenced the level of cariogenic activity within the sample. Children attending the program more frequently (>19 appointments) had a greater mean number of inactivations at the end of the study compared to those with low attendance (< 19 appointments; $\mathrm{P}=0.005$ ).

The mean percentage of CD4 was $23.83 \%$ ( $\mathrm{SD}=$ $12.02)$ at the baseline and $21.63 \%$ (S.D. $=10.16)$ at the end of the study $(\mathrm{p}>0.05)$.

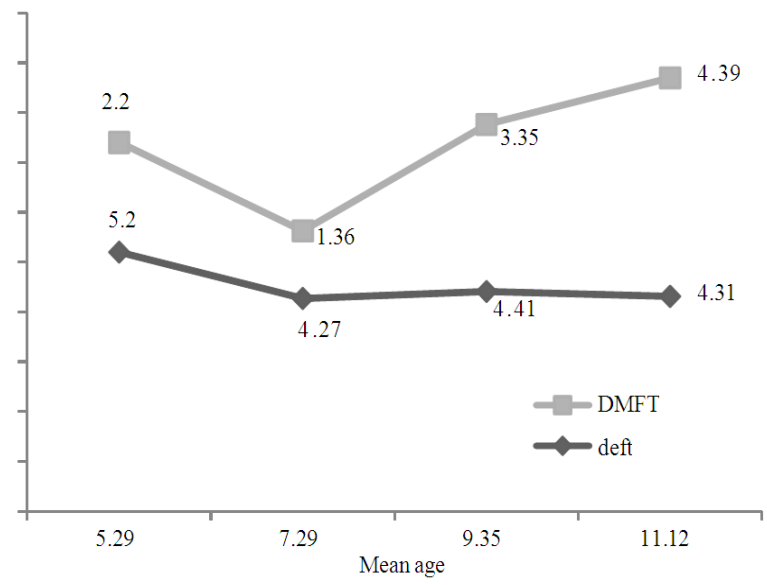

Fig. 2: Caries indexes (modified deft/DMFT) at baseline, 2 years, 4 years and at end of evaluation of the oral health-promoting program for HIV-infected children

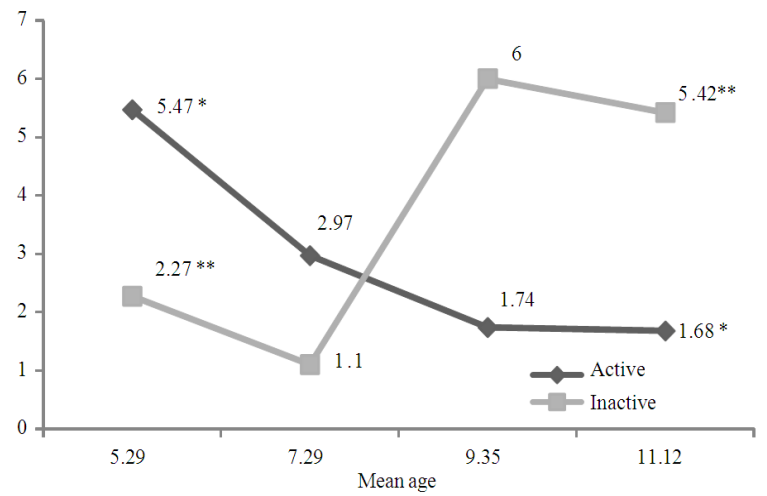

Fig. 3: Mean number of active and inactive carious lesions at baseline, 2 years, 4 years and at end of evaluation of the oral health-promoting program for HIV-infected children (MannWhitney test: *, P <0.005; **, P <0.001) 
Am. J. Infect. Dis., 8 (2): 73-78, 2012

Table1: Caries indexes (modified deft/DMFT) and frequency of carious lesions at baseline and final evaluation of the oral health promoting program for HIV-infected children

\begin{tabular}{|c|c|c|c|c|c|}
\hline \multirow[b]{2}{*}{ Caries indexes } & \multicolumn{2}{|c|}{ Baseline } & \multicolumn{2}{|c|}{ Final } & \multirow[b]{2}{*}{ p-value } \\
\hline & $\mathrm{n}$ & Mean (SD) & $\mathrm{n}$ & Mean (SD) & \\
\hline$\overline{\text { Deft }}$ & 30 & $5.20(4.27)$ & 16 & $4.31(3.59)$ & $>0.05$ \\
\hline Defs & 30 & $9.40(9.35)$ & 16 & $8.62(9.17)$ & $>0.05$ \\
\hline DMFT & 15 & $2.20(1.97)$ & 31 & $4.39(3.31)$ & $>0.05$ \\
\hline DMFS & 15 & $4.20(5.07)$ & 31 & $6.29(5.29)$ & $>0.05$ \\
\hline Carious lesions & 31 & $7.73(6.41)$ & 30 & $7.41(5.46)$ & $>0.05$ \\
\hline
\end{tabular}

Table 2: Mean number of active and inactive carious lesions in relation to immunosuppression at the end of evaluation

\begin{tabular}{|c|c|c|c|c|}
\hline \multirow{2}{*}{$\begin{array}{l}\text { Caries indexes } \\
\text { (final) }\end{array}$} & \multicolumn{2}{|c|}{ Severe immunosuppression } & \multicolumn{2}{|c|}{ Moderate immunosuppression/Absent } \\
\hline & $\mathrm{n}$ & Mean (SD) & $\mathrm{n}$ & Mean (SD) \\
\hline Active caries & 9 & $1.22(2.38)$ & 17 & $0.88(1.21)$ \\
\hline Inactive caries & 9 & $4.33(3.20)$ & 17 & $5.41(4.54)$ \\
\hline
\end{tabular}

At final evaluation, no relationship was found between presence of active and inactive caries in relation to immunosuppression, as similar results regarding active and inactive caries were obtained in severely immunosuppressed patients compared to those less severely affected (Table 2).

\section{DISCUSSION}

Implementing oral and dental health services is of great importance for pediatric patients infected by HIV. However, there is very little information about such availability in the literature (Hastreiter and Jiang, 2002). Such studies, therefore, are highly relevant for those practitioners providing these services. In a group of healthy children, promissory results were observed after an implemention of a dental caries prevention program in a clinic serving low-income residents. After 26 months, it was observed a reduce in dental caries experience in this population (Minah et al., 2008). Another study, observed that the mean dental plaque and gingival bleeding scores gradually increased after interruption of the educational activities of an oralhealth-promotion program, verifying that the duration of the program favorably influenced its outcome (Antonio et al., 2007).

In this study, analysis of the caries indexes showed that the presence of lesions in both deciduous and permanent dentitions of HIV-infected children is high. However, these results are better than those obtained in a Brazilian epidemiological survey, showing mean deft value of 2.80 for a 5-year age group and a mean DMFT value of 2.78 for a 12-year age group (Saude, 2004). In addition, the national program cited above did not take into account active caries, which might have underestimated the disease.

Considering that the program was implemented during the mixed dentition, the caries indexes should be evaluated as being relatively stable, mainly in relation to the deciduous dentition. The small reduction in deft and defs indexes may be attributed to dental exfoliation, whereas the permanent dentition had marked increases in DMFT (100\%) and DMFS $(50 \%)$ despite the lack of a statistically significant difference. Also, the increased number of permanent teeth, which are more susceptible to etiological causes of caries, should be also be considered. These results are clearly observed when the evolution of caries indexes are analyzed according to the age group of patients in the program. On the other hand, Hicks et al. (2000) in a longitudinal study of HIV-infected children between 2 and 9 and found that the defs values increased over a period of 30 months, thus resulting in a reduction in the number of caries-free children. Although these children did not participate in an oral health program, the difference regarding evaluation time limits any comparison between the studies.

In a 1-year intensive program for HIV-infected children (mean age 5.8), whose main objectives were to provide hygiene instructions and to remove biofilm every month, Ribeiro et al. (2007) observed that the high caries indexes (defs 16.77; DMFS 5.41) did not change at the end of evaluation. However, the mean amount of dental surface with active caries decreased from 8.03-5.80, whereas inactive caries increased from 3.76-5.91 (Ribeiro et al., 2007). Despite the fact that the intensive program lasted only 1 year, the authors used an indicator of caries activity, which was the most reliable approach for detecting the program's efficacy in controlling this disease. According to the authors, both individual care and rigorous follow-up of oral hygiene led to good results.

In the present study, teeth were used for evaluating caries activity and the results were similar to those found by Ribeiro et al. (2007), as the amount of inactive caries increased significantly while active caries decreased, thus demonstrating the efficacy of the 
program. In our program, children are also individually monitored and have their toothbrushing supervised by an instructor at the medical outpatient clinic every month. In addition, their caregivers are instructed about oral health care and diet. All these procedures seem to help control the caries. According to Hilton et al. (2007), an efficient oral health preventive program should include educational interventions aiming to provide oral hygiene practices, adequate diet and healthy habits in addition to encouraging early professional treatment.

It was also observed that more frequent monitoring (> 19 appointments) was more effective in inactivating caries. These results should be valued not only because HIV-infected children may be considered at high caries risk, but also because it is a long-term study. The importance of oral health services has also been observed for HIV-infected adults, demonstrating the effectiveness of primary care for adults as regular patients (Hastreiter and Jiang, 2002). Also to be considered is that the program was idealized for following up children and their caregivers on the same day as the medical consultation, thus reducing travel costs for these poor families. This same-day scheduling of dental and medical appointments can explain the variation in the number of consultation visits observed over the study period.

Moreover, providing consultation within the medical outpatient unit allows patient and caregiver to see that the surgeon-dentist is part of a multidisciplinary caregiving team. Unfortunately, many clinical services do not follow this approach, resulting in oral health care that may be compromised by the lack of specialization. According to Ramos-Gomez et al. (2000), it is essential that developing countries provide their health care professionals with preventive training so that the identification and treatment of orofacial manifestations of HIV can be properly made, especially in the case of pediatric patients.

Vieira et al. (1998), who compared caries indexes of HIV-positive immunosuppressed children $(\mathrm{T} 4 / \mathrm{T} 8<0.5)$ with those of nonimmunosuppressed ones $(\mathrm{T} 4 / \mathrm{T} 8>0.5)$, found that the former had higher caries indexes in both dentitions. These results, however, were not observed in the present study as the difference between active and inactive caries in our sample was not found to be statistically significant. On the other hand, Hicks et al. (2000) and Castro et al. (2004) have observed that children with low levels of CD4 had higher defs index. In the present study, the number of severely immunosuppressed patients was small, thus demonstrating that most children were immunologically healthy as their mean CD4 count was relatively high $(19.85 \%)$.
It is important to emphasize that promoting oral health among these patients is not limited to controlling the emergence and progress of caries. Immunodepressed individuals are more susceptible to infections, particularly candidiasis, which is the most common opportunistic infection in HIV-infected children (Ramos-Gomez et al., 2000; Ramos-Gomez, 2002; Olaniyi and Sunday, 2005). Studies have shown a relationship between dentinal caries and prevalence of Candida species (spp), suggesting that such lesions can serve as reservoirs for yeasts-a risk factor for oral colonization and infection (Cerqueira et al., 2007; Rego et al., 2003; Starr et al., 2002). An association between fungal infection and higher caries index in HIVinfected children was also observed (Chen et al., 2003). All these findings further support the fact that such individuals need ongoing oral health care.

Caries prevention and control represent a great challenge for this population; other risk factors besides the traditional ones may be involved, such as continued use of saccharin-containing medications, lack of specific monitoring and peculiar immunological aspects (e.g. local and systemic immune-deficiency) regarding HIV infection are involved (Madigan et al., 1996; Valdez et al., 1994; Castro et al., 2004).

\section{CONCLUSION}

The implementation of oral health-promoting measures is, therefore, fully justified in preventing caries in HIV-infected children. Measures of this kind will contribute to improving the prognosis of systemic diseases as well as the quality of life of these children.

\section{REFERENCES}

Antonio, A.G., A. Kelly, D.D. Valle, R.B. Vianna and L.E. Quintanilha, 2007. Long-term effect of an oral health promotion program for schoolchildren after the interruption of educational activities. J. Clin. Pediatr. Dent., 32: 37-41. PMID: 18274468

Bjorndal, L., T. Larsen and A. Thylstrup, 1997. A clinical and microbiological study of deep carious lesions during stepwise excavation using long treatment intervals. Caries Res., 31: 411-417. PMID: 9353579

Carvalho, J.C., Thylstrup, A., Ekstrand, K.R., 1992. Results after 3 years of non-operative occlusal caries treatment of erupting permanent first molars. Community Dent. Oral Epidemiol., 20: 187-192. PMID: 1526101

Castro, G.F., I.P. Souza, S. Lopes, P. Stashenko and R.P. Teles, 2004. Salivary IgA to cariogenic bacteria in HIV-positive children and its correlation with caries prevalence and levels of cariogenic microorganisms. Oral Microbiol. Immunol., 19: 281-288. PMID: 15327638 
CDCP, 1994. 1994 Revised classification system for human immunodeficiency virus infection in children less than 13 years of age. Morb. Mortal. Wkly. Rep. (MMWR), 43: 1-19.

Cerqueira, D.F., M.B. Portela, L. Pomarico, R.M.A. Soares and I.P.R. Souza, 2007. Examining dentinal carious lesions as a predisposing factors for the oral prevalence of Candida spp in HIV-infected children. J. Dent. Child. (Chic), 74: 98-103. PMID: 18477427

Chen, J.W., C.M. Flaitz, B. Wullbrandt and J. Sexton, 2003. Association of dental health parameters with oral lesion prevalence in human immunodeficency virus-infected Romanian children. J. Pediatr. Dent., 25: 479-484. PMID: 14649612

Exposito-Delgado, A.J., E. Vallejo-Bolanos and E.G. Martos-Cobo, 2004. Oral manifestations of HIV infection in infants: A review article. Med. Oral. Patol. Oral. Cir. Bucal., 9: 415-420. PMID: 15580118

Gelbier, M., V.S. Lucas, N.E. Zervou, G.J. Roberts and V. Novelli, 2000. A preliminary investigation of dental disease in children with HIV infection. Int. J. Paediatr. Dent., 10: 13-18. PMID: 11310121

Hastreiter, R.J. and P. Jiang, 2002. Do regular dental visits affect the oral health care provided to people with HIV? J. Am. Dent. Assoc., 133: 1343-1350. PMID: 12403536

Hicks, M.J., C.M. Flaitz, A.B. Carter, S.G. Cron and S.N. Rossmann et al., 2000. Dental caries in HIVinfected children: A longitudinal study. Pediatr. Dent., 22: 359-364. PMID: 11048301

Hilton, I.V., S. Stephen, J.C. Barker and J.A. Weintraub, 2007. Cultural factors and children's oral health care: A qualitative study of carers of young children. Community Dent. Oral Epidemiol., 35: 429-438. PMID: 18039284

Madigan, A., P.A. Murry, M. Houpt, F. Catalanotto and M. Feuerman, 1996. Caries experience and cariogenic markers in HIV-positive children and their siblings. Pediatr. Dent., 18: 129-136. PMID: 8710715

Maguire, A., A.J. Rugg-Gunn and T.J. Butler, 1996. Dental health of children taking antimicrobial and non-antimicrobial liquid oral medication long-term. Caries Res., 30: 16-21. PMID: 8850578

Minah, G., C. Lin, S. Coors, I. Rambob and N. Tinanoff et al., 2008. Evaluation of an early childhood caries prevention program at an urban pediatric clinic. Pediatr. Dent., 30: 499-504. PMID: 19186776

Olaniyi, T.O. and P. Sunday, 2005. Oral manifestations of HIV infection in 36 Nigerian children. J. Clin. Pediatr. Dent., 30: 89-92. PMID: 16302607
Phantumvanit, P. and T. Pilot, 1994. Atraumatic Restorative Treatment Technique of Dental Caries. 2nd Ed. World Health Organization, Geneva, pp: 52.

Ramos-Gomez, F., 2002. Dental considerations for the paediatric AIDS/HIV patient. Oral Dis., 2: 49-54. PMID: 12164660

Ramos-Gomez, F.J., A. Petru, J.F. Hilton, A.J. Canchola and D. Wara, et al., 2000. Oral manifestations and dental status in paediatric HIV infection. Int. J. Paediatr. Dent., 10: 3-11. PMID: 11310124

Rego, M.A., C.Y. Koga-Ito and A.O. Jorge, 2003. Effects of oral environment stabilization procedures on counts of Candida spp. in children. Pesqui. Odontol. Bras., 17: 332-336. PMID: 15107915

Ribeiro, A.A., E.R. Bundzman, C. Nishio and I.P.R. Souza, 1998. Relation between salivary flow, medicines and caries in HIV+ children. J. Dent. Res., 77: 841-841.

Ribeiro, A.A., M.B. Portela and I.P.R. Souza, 2007. Evaluation of an oral health promotion program in HIV-positive children. Caries Res., 41: 269-269.

Saúde, M.D., 2011. Boletim Epidemiológico AIDSDST.

Saude, S.D.A., 2004. Condições de saúde bucal da população brasileira 2002-2003: Resultados principais.

Starr, J.R., T.C. White, B.G. Leroux, H.S. Luis and M. Bernardo et al., 2002. Persistence of oral Candida albicans carriage in healthy Portuguese schoolchildren followed for 3 years. Oral Microbiol. Immunol., 17: 304-310. PMID: 12354212

Tofsky, N., E.M. Nelson, R.N. Lopez, F.A. Catalnotto and D.H. Fine et al., 2000. Dental caries in HIVinfected children versus household peers: Two-year findings. Pediatr. Dent., 22: 207-214. PMID: 10846731

WHO, 2006. AIDS Epidemic Update, December 2006. 1st Edn., World Health Organization, Geneva, ISBN-10: 9291735426, pp: 86.

Valdez, I.H., P.A. Pizzo and J.C. Atkinson, 1994. Oral health of pediatric AIDS patients: a hospital-based study. ASDC J. Dent. Child., 61: 114-118. PMID: 8046089

Vieira, A.R., I.P.D. Souza, A. Modesto, G.F. Castro and R. Vianna, 1998. Gingival status of HIV+ children and the correlation with caries incidence and immunologic profile. Pediatr. Dent., 20: 69-172. PMID: 9635311

WHO, 1997. Oral Health Surveys: Basic Methods. 4th Edn., World Health Organization, Geneva, ISBN10: 9241544937, pp: 66. 